\title{
The exciting field of neuro-repair in multiple sclerosis: an interview with Sarah I Sheikh
}

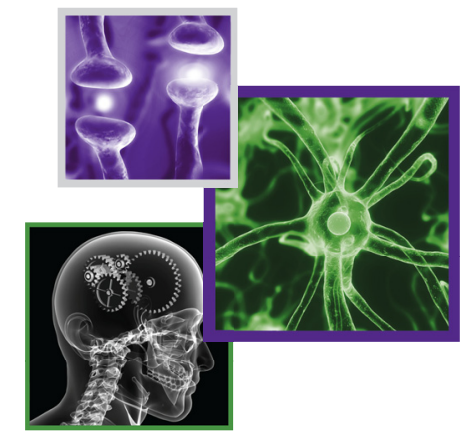

Sarah I Sheikh*,1

${ }^{1}$ Senior Medical Director, Late Stage Clinical Development at Biogen, Cambridge, MA 02142, USA

* Author for correspondence: sarah.sheikh@biogen.com

Sarah Isabel Sheikh* speaks to Laura Dormer, Commissioning Editor: Sarah I Sheikh, MD, MSc, MRCP, is a Senior Medical Director in Late Stage Clinical Development at Biogen. Her current focus is on developing therapies for multiple sclerosis/neuroinflammation, remyelination and neuro-repair. Prior to Biogen, Dr Sheikh was an attending in Neurology at Brigham and Women's Hospital. She completed her internship at Massachusetts General Hospital and residency in Neurology and Neuromuscular fellowship at Massachusetts General Hospital and Brigham and Women's Hospital. She received her medical degree from Oxford University Medical School, and a Masters in cell physiology from Oxford University, Corpus Christi College. She is a member of the Royal College of Physicians, London.

Accepted for publication: 17 November 2017; Published online: 31 January 2018

Please could you introduce yourself, \& tell us about your role at Biogen?

I lead the late-stage multiple sclerosis (MS) and neuro-repair group here at Biogen. I have been here for 7 years, and am a neurologist by training.

\section{Could you briefly summarize the Phase II SYNERGY trial \& its findings?}

The opicinumab molecule is a monoclonal antibody against LINGO-1, and it is thought that blocking LINGO-1 will aid remyelination by allowing oligodendrocyte precursor cells to differentiate and be involved in neuro-repair. The SYNERGY Phase II study was a 72-week, randomized, placebo-controlled, dose-finding study, which included four doses of opicinumab versus placebo. The doses explored were 3, 10, 30 and $100 \mathrm{mg} / \mathrm{kg}$. Opicinumab was given as an add-on therapy to patients who were concurrently taking intramuscular IFN- $\beta-1 \mathrm{a}$.

Very briefly, the key findings were of an inverted U-shaped dose-response, where the 10 - and $30-\mathrm{mg} / \mathrm{kg}$ dose groups appeared to do better. In particular, the $10-\mathrm{mg} / \mathrm{kg}$ dose really stood out, across multiple end points and sensitivity analyses. But overall, that study was negative because what had been assumed had been a monotonic linear dose-response, rather than the inverted U-shape that was seen. What we did see for the $10-\mathrm{mg} / \mathrm{kg}$ dose group, though, was tantalizing. This led us to try and understand these data better. It is important to remember that nobody has ever achieved repair of the CNS in humans, and so we are breaking new ground as we do these studies, and try to understand how we can impact neuro-repair. So we considered what could be learned from the data in this study, in order to continue our evaluation of this field and move it forward to potentially benefit patients.

You are lead author on the SYNERGY post hoc analysis poster presented at the recent ECTRIMS-ACTRIMS conference [1], which identified a subgroup of patients who may benefit more from opicinumab treatment. What were the characteristics of this subpopulation? The data presented at ECTRIMS were a post hoc analysis, using a systematic multivariate approach to look for those attributes which were most predictive of a positive treatment response. All baseline characteristics, including baseline demographics and MRI characteristics, were put into a model to compete with each other. This analysis uncovered three baseline factors that predicted higher efficacy of opicinumab; disease duration $\leq 20$ years, and two MRI factors - diffusion tensor imaging (DTI) in T2 lesions and magnetization transfer ratio (MTR) in T2 lesions. DTI is reasonably reflective of relative tissue integrity in that lesion. MTR can be thought of as corresponding to myelin content in the lesion. When you put that together, what it suggests is that the patients identified to have a potentially better response to opicinumab are those who have lesions that have relative structural integrity, but have lower myelin. So there was biologic plausibility to this, given that the lesion characteristics were suggestive of

Future $\because \cdots$ Medicine 
a substrate that may be amenable to repair with a remyelinating therapy. Of note, a subset of of around 25-30\% of the SYNERGY population met these post hoc analysis criteria.

Taken together, this means that we think we have identified a dose, the $10-\mathrm{mg} / \mathrm{kg}$ dose, which appeared to do best in the Phase II SYNERGY study, and a population in whom we can take this molecule forward for potential benefit in MS. That is what the next study, the AFFINITY study, is based on.

\section{What results were seen in this subgroup of patients?}

Within the post hoc analysis subgroup of patients, we saw increased efficacy that was sustained over time. This was assessed using an integrated measure of disability which includes improvement and worsening over time, called the overall response score. Its components are the Expanded Disability Status Scale, timed 25-foot walk and 9-hole peg test. This is also what we will be using as the primary end point in the AFFINITY study.

\section{What are the aims of the AFFINITY study?}

The AFFINITY study was really born out of the learnings from the SYNERGY study, and is a recently initiated Phase II study aiming to enroll around 240 patients with relapsing MS. It is a placebo-controlled, double-blind study, using opicinumab as an add-on therapy in patients who are reasonably well-controlled on their background anti-inflammatory disease-modifying therapies.

This study will broaden the aperture of the previous study; whereas in SYNERGY we used IFN- $\beta-1$ a as the background therapy, here there will be patients on interferons, dimethyl fumarate and natalizumab, reflecting different mechanisms of action, different modes of administration and different levels of anti-inflammatory effect. And of course, the study population is very clearly defined by the criteria we found in the post hoc analysis (of the SYNERGY study), so will include patients with DTI and MTR in the T2 lesions, and disease duration of less than or equal to 20 years, along with an Expanded Disability Status Scale between 2 and 6. Also following on from SYNERGY, we will be using the novel end point mentioned previously, the overall response score, as our primary end point. The study will be 72 weeks in duration, and will use a dose based on the $10-\mathrm{mg} / \mathrm{kg}$ dose identified previously, but this time using a fixed dose of $750 \mathrm{mg}$, once every 4 weeks as an intravenous infusion.

The interesting thing here is that no one has ever done a study enrolling patients based on these types of imaging criteria, so again we are entering new ground with biologic plausibility to it. It is very exciting to be a part of that, to learn from the previous study and all the work that has been done, and try to move it forward. We recently enrolled our first patient in the study!

\section{How is this new data significant for MS patients?}

As you can imagine, there is a lot of excitement in the MS community about a study like this, because we are investigating an add-on therapy, and one that offers the potential of neuro-repair and functional improvement. Everyone is very excited about that. Also, by particularly targeting a certain subgroup of patients we are moving the field forward into more of a precision medicine approach.

\section{Looking forward, can you elaborate on Biogen's main areas of research focus in this area?}

From our perspective, focusing on these subpopulations makes the most sense from what has been seen in the previous trial - to learn from the existing data and use those learnings to build upon. We are dedicated to the field of neuro-repair, and applying those learnings to future endeavors is going to be critical.

As a company, Biogen has been committed to the field of MS for over 20 years, and that commitment remains very strong, and a key pillar of what we do. We are always looking at new ways to treat MS and to improve disability, and I think that is the key thing about the AFFINITY study - we are looking to improve pre-existing disability, whereas the anti-inflammatory medications that we have in MS currently try and slow down disability that may occur in the future. Looking to repair or improve existing disability is quite a different concept, and an inspiring one. If that worked, it would really change the field. As I mentioned at the start, no one has ever achieved repair of the human CNS, so it is a daunting but exciting task, that is important for patients - improving their function, improving how they feel and ameliorating such a devastating disease.

Ultimately, our focus at Biogen is on a continued commitment to MS, bringing our experience to bear as we move the field further, working in the key areas that are still unmet despite all the advances in the field. Our goal is to develop even more effective therapies, improve disability and achieve neuro-repair; but most importantly staying committed to the cause of the patients. 


\section{Acknowledgements}

The opinions expressed in this interview are those of SI Sheikh and do not necessarily reflect the views of Future Medicine Ltd.

Financial \& competing interests disclosure

SI Sheikh is a full-time employee of Biogen. The interviewee has no other relevant affiliations or financial involvement with any organization or entity with a financial interest in or financial conflict with the subject matter or materials discussed in the manuscript apart from those disclosed.

No writing assistance was utilized in the production of this manuscript.

\section{References}

1. Sheikh S, Calabresi PA, Giovannoni G et al. Predictors of an opicinumab treatment effect and identification of an efficacy subpopulation: a post hoc analysis of the SYNERGY study. Presented at: 7th Joint ECTRIMS-ACTRIMS Meeting. Paris, France, 25-28 October 2017 (Abstract P718). 
